Review Article

\title{
A literature review on multimodality molecular imaging nanoprobes for cancer detection
}

\author{
Daryoush ShaHBAZI-GaHROUEI ${ }^{1, a}$, Pegah MoRADI KHANIABADI ${ }^{2}$, Saghar SHAHBAZI-GAHROUEI ${ }^{1}$, Amir \\ KHORASANI ${ }^{1}$, Farshid MAHMOUDI ${ }^{1}$ \\ ${ }^{I}$ Dept. of Medical Physics, School of Medicine, Isfahan University of Medical Sciences, Isfahan, Iran \\ ${ }^{2}$ School of Physics, Universiti Sains Malaysial 1800, Pulau Penang, Malaysia \\ ${ }^{a}$ E-mail address: shahbazi@med.mui.ac.ir
}

(received 25 December 2018; revised 14 February 2019; accepted 26 March 2019)

\begin{abstract}
Molecular imaging techniques using nanoparticles have significant potential to be widely used for the detection of various types of cancers. Nowadays, there has been an increased focus on developing novel nanoprobes as molecular imaging contrast enhancement agents in nanobiomedicine. The purpose of this review article is to summarize the use of a variety of nanoprobes and their current achievements in accurate cancer imaging and effective treatment. Nanoprobes are rapidly becoming potential tools for cancer diagnosis by using novel molecular imaging modalities such as Ultrasound (US) imaging, Computerized Tomography (CT), Single Photon Emission Tomography (SPECT) and Positron Emission Tomography (PET), Magnetic Resonance Imaging (MRI), and Optical Imaging. These imaging modalities may facilitate earlier and more accurate diagnosis and staging the most of cancers.
\end{abstract}

Key words: molecular imaging; nanoprobes; cancer; nanobiomedicine.

\section{Introduction}

Multimodality imaging is an emerging subject that integrates advanced imaging technology with cellular and molecular biology. Multimodality molecular imaging has the strength to enhance all features of cancer care [1]. High resolution and highly sensitive systems and specific imaging contrast agents (CAs) are necessary to enhance the imaging resolution with a molecular event. Tumor characterization without invasive procedures such as biopsies or surgery is considered another key feature molecular imaging adds to cancer diagnosis [2].

Imaging modalities require high resolution in addition to high sensitivity features to detect and recognize specific imaging agents that link the imaging signal with molecular size. It may consider tumor characterization and cancer diagnosis without any invasive operations like biopsy or even surgery [3]. There are many types of imaging contrast agents such as small molecules, peptides, aptamers, high-molecularweight antibodies, and various nanoparticles [3-6].

Four imaging modalities including, computed tomography (CT), magnetic resonance imaging (MRI), single-photon emission computed tomography (SPECT) and positron emission tomography (PET), are widely used for threedimensional imaging in cancer staging [7]. All mentioned imaging modalities suffer from deficiencies in sensitivity and resolution. With the possibility to solve several imaging diagnostic problems in terms of scale and to enhance diagnosis ability; they were not designed to image small numbers of cancer cells [8]. The aim of cancer imaging should be to detect the smallest possible number of cancer cells before the angiogenesis. In recent years, nanoprobes have attracted significant attention as contrast agents for early detection of cancer in molecular imaging. Using nanoprobes, delivering a large payload quantity in a single dose, achieving great specificity toward a target and, carrying both imaging agents and therapeutic drugs is possible [9].

Generally speaking, phenotypic, targeted cell-tracking and reporter gene probes are the main probes for molecular imaging. The phenotypic probe is utilized to determine general features of malignant physiology, such as angiogenesis, cell proliferation, apoptosis, and the expression of certain receptors in cancer cells. Targeted cell-tracking is employed to image specific molecules which are characteristics of a tumor.

In this article, the current state of the art, recent findings, and advanced approaches in multimodality molecular imaging using novel nanoprobes for cancer detection are considered.

\section{Ultrasound Molecular Imaging}

Ultrasound (US) imaging is a non-invasive imaging modality with a high soft tissue contrast and without exposing the patient to radiation. For this reason, clinical US imaging is widely used. It has been used to classify benign, solid lesions with a negative predictive value of $99.5 \%$ [10] and can be applied for 
both imaging and therapeutic purposes. Li et al. [11] used multi-targeted tyrosine kinase inhibitor Lenvatinib which covalently bound with the superparamagnetic iron oxide nanoparticles (SPIONs) to improve US image quality. They have reported that contrast-enhanced ultrasound improved the imaging quality and diagnostic accuracy of an early stage of non-small cell lung cancer (NSCLC).

In this imaging modality, different types of bubbles are used as imaging contrast agents with sizes of nano- to micro- meters [12]. Microbubble (MB) as a new contrast agent can enhance the specificity and sensitivity of US imaging for cancer diagnosis.

Beik et al. [13] used the gold nanoparticle (AuNP) as an ultrasound nanosonosensitizer agent for colon tumor detection in mice. Their findings have supported the use of AuNP as a potent sonosensitizing agent for both thermal and mechanical effects of ultrasound.

Ultrasound is able to expose the flow dynamic of vessels lesser than $500 \mu \mathrm{m}$ which is greater than angiogenic capillaries using targeted microbubbles. A study in molecular biology has proved that new angiogenic biomarkers with the use of US imaging contrast agent enabled the specific and sensitive representation of molecular targets [14].

Martins et al. [15] suggested that microbubble targeting vascular endothelial growth factor receptor (VEGFR-2), a receptor tyrosine kinase using US imaging, might be as a potential method for detecting VEGFR-2 expression in colorectal cancer.

Recently, Abu-Elkacem et al. [16] introduced a novel molecular targeted MB conjugated with the 10th type of human-fibronectin. According to their findings, VEGFR-2 was imaged on the neovasculature of breast cancer. Moreover, Hao and co-workers [17] developed high molecular polymer $\mathrm{MB}$ which marked by quantum dots (MBQDs/PLGA/Her). They have confirmed the diagnosis and treatment of ovarian cancer by both US imaging and photothermal therapy. In another work, Lutz et al. [18] developed a mouse model that might make easy the pre-clinical development of microbubbles targeted human vascular.

\section{SS-MB}

\section{no US}
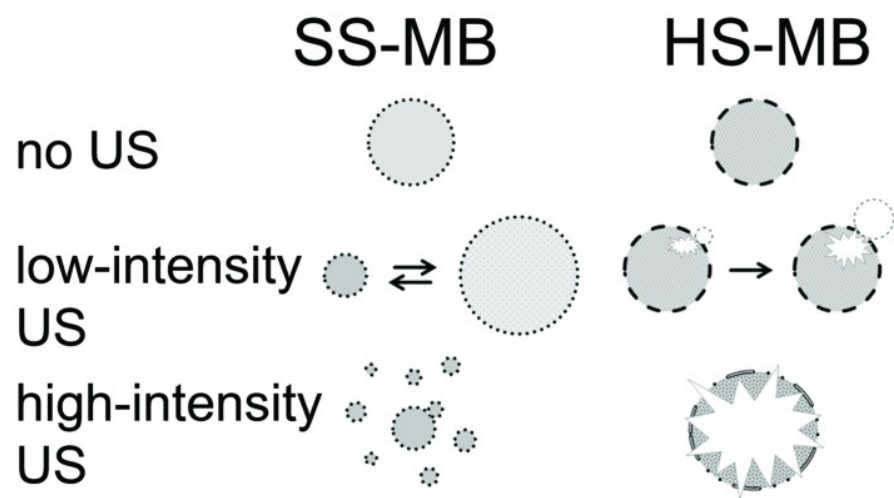

Figure 1. Behavior of SS-MB and HS-MB at different ultrasound intensities [20].
Furthermore, for clinical features of US imaging, MBs are conjugated with genes or drugs specifically used for targeted therapy. On the other hand, targeted MB in US imaging may enhance the permeability of the cellular membrane by creating pores in the cell membrane (sonoporation). Hence, targeted microbubble conjugated with drugs can treat cancer. Recently, Smart gold nanoparticle-stabilized microbubbles (SAuMBs) have been used as theranostic ultrasound agents. SAuMBs composed of a gas-filled core and shell including SAuNPs which can be aggregated in tumors by sonoporation. Researchers have been shown, the aggregation of SAUNPs offering a way for photoacoustic (PA) monitoring and photothermal treatment (PPT) of the tumor [19].

So far, nano-sized bubbles with various shells consisting of different specific polymers have been applied in extravascular US imaging. Soft-shell microbubbles (SS-MB) are commonly used with non-linear oscillations ultrasound. The better oscillation properties of SS-MB compared with hard-shell microbubbles (HS-MB) are due to the thinner layer and more flexible shells, which are held together not only by covalent bonding but also by hydrophobic interactions [20]. However, after slight shell disruptions, the shell seals itself to minimize surface tension. Using high acoustic pressure can be caused the MB split into several smaller bubbles instead of bursting like HS-MB as it was illustrated in Figure 1. To achieve the best contrast function, the shells' features should be considered following by adjustment acoustic power to the type of MB.

One strategy for cancer detection in US imaging is the application of specific antibody conjugated with MB targeting ligands. Many research studies have used this strategy for the observation of different type of cancers. Yildirim et al. [21] reported an ablation technique which induces mechanical damage rather than thermal damage at lower acoustic pressures by utilizing phospholipid-stabilized hydrophobic mesoporous silica nanoparticles (PL-hMSN). McLaughlan et al. [22] used plasmonic gold nanorods (AuNR) as theranostic agent in high intensity focused ultrasound (HIFU) for the treatment of cancerous tissue.

It is important to know that among many new techniques, breast ultrasound provides useful information such as lymph nodes in the axilla between the pectoral muscles, and in the subclavian region, the neck and the medial thoracic chain. Also, the flexibility of US imaging is superior to those of other modalities, in case of taking biopsy, follow-up, and fusion.

\section{Computed Tomography Scanning (CT-Scan)}

Computed tomography is a potent diagnostic imaging modality that is low expensive, deep tissue permeation, great spatial and high resolution. However, low signal-to-noise ratio reduced the ability of CT to distinguish between neighboring tissues [1]. This disadvantage was compensated by applying contrast media which have chemical stability, high solubility, denseness, and toxicity. The integral role of molecular imaging 
is to match the traditional imaging technique modalities, using micro or nanoprobes which enhance the detections of cellular events in addition to early detection of different types of cancers.

Several heavy atoms such as iodine, tungsten, and barium are suitable as contrast media in CT images due to great $\mathrm{x}$-ray attenuation coefficient. For example, Sevda Nedaei et al. [23] prepared PEGylated melanin nanoparticles (MNPs) reacted with barium ions as CT contrast media (Ba-MNPs-mPEG). Wallyn and co-workers applied iodinated polymeric nanoparticles with strong X-ray attenuation capacity in the liver and spleen [24]. Similarly, a new polymer (triiodobenzene) iodine nanoparticle (INP) with a PEG coating was designed as a contrast agent for tumor loading by Hainfeld et al. [25]. The iodine-based CT contrasts are either monomeric or dimeric forms of 1,3,5-triiodobenzene derivatives [26]. CT always merges with other modalities for anatomical imaging such as MRI [27], PET [28] as well as molecular imaging to enhance functional imaging and to reflect essentially anatomical conversion by employing novelties beacons that detect cellular events.

It was reported that contrast media which contain multiple contrast elements and multiple K-edge values, making their contrast efficiencies well tailored to the operating voltage, and thus resulting in an optimal CT contrast efficiency when used for various patients (with different age, gender, and weight). In this regard, Zheng et al. [29] introduced a novel binary CT contrast medium which had two kinds of CT contrast ions $\left(\mathrm{Ba}^{2+}\right.$ and $\left.\mathrm{Ho}^{3+}\right)$ were co-doped into nano-hydroxyapatite (nHAp, the main inorganic component of human bone and teeth) host. They have indicated that $\mathrm{Ba} / \mathrm{Ho}$ co-doped nHAp greatly enhanced CT contrast efficiency which could be ascribed to the strong X-ray adsorption ability of $\mathrm{Ba}^{2+}$ and $\mathrm{Ho}^{3+}$ co-existence. In another study, Naha et al. [30] proved that the utilization of gold-silver alloy nanoparticle can be used as contrast media for CT and dual-energy mammography. They found that novel contrast media might be useful for screening breast cancer and blood pool.

Generally, there are three major applications of nanoparticles which utilize as X-ray contrast media in diagnosis; (a) blood pool, (b) passive targeting, and (c) active targeting. Shi et al. [31] used Au(III) ions to fabricate a novel dendrimer-stabilized gold nanoparticle for imaging and targeting cancer cells. They found that Au-DSNPs targeted the cells that expressed folic acid and fluorescein isothiocyanate. A year later, Eck et al. [32] introduced CT contrast media which targeted CD4 receptor of peripheral lymph nodes conjugated gold nanoparticles.

Furthermore, Zhang et al. [33] evaluated AuNPs for imaging of microdamage in bone tissue using a conventional method. In another work, Aydogan et al. [34] conjugated gold nanoparticles and 2-deoxy-D-glucose. They reported that by using a micro CT scanner, a novel fabricated CT contrast media was able to detect human alveolar epithelial cancer invitro. Recently, a new cost-effective CT contrast media has been designed and targeted overexpressing folic acid side of cancer cells by utilizing CT imaging modality. The fabricated folic acid targeted polyethlenimine-entrapped AuNPs showed potential CT contrast in both in vivo and in vitro studies [35]. In a similar work, Khademi et al. [36] reported a simple method of forming folic acid-targeted multifunctional gold nanoparticles via cost-effective cysteamine as a template for molecular CT imaging of human nasopharyngeal epidermoid carcinoma cells in vitro. Additionally, it was reported elsewhere that Au-PSNPs (methyl-orange-doped polystyrene gold nanoparticle) not only confirmed as CT contrast media but also suggested that it can be utilized for blood pool imaging [37]. The slight difference in the size of AuNPs after modification is due to either specific localization of gold nanoparticle in targeted tumor [38]. Moreover, modification of the surface of AuNPs by coating with polymers such as glycol chitosan or other chemical compound is affected the biological behavior of NPs at the target sides and increases the stability of nanoparticles, thus improving imaging capability $[39,40]$.

It was reported the unique biological properties of CT contrast media, Au-DENPs (Au-dendrimer-entrapped gold nanoparticle) with a neutral surface for SPC-A1 in-vitro as well as the xenograft tumor model [41]. Moreover, Au-DENPs-FA was presented as a potential CT contrast media for human lung adenocarcinoma [17].

To increase the practicality and the accuracy of tumor imaging, dual modality is necessary. For example, Au-DENPs conjugated gadolinium and M-NPAPF-Au demonstrated as great specific nanoprobes to study the internal organs of a mouse for CT/MRI and fluorescence/CT imaging, respectively [42]. Huiting et al. [43] introduced Bismuth (Bi) nanoparticles as another dual-modality imaging contrast agent for fluorescence/CT imaging of liver and intestines and tracing of the tumor metastasis. They have shown that Bi nanoparticles can create high CT contrast image and upon the light irradiation, it can emit luminescence photons with diverse color from purple to red. Furthermore, when the wavelength of excitation light was larger than $450 \mathrm{~nm}$, the sample emitted strong near-infrared light, which can be used for NIR imaging.

Using cancer biomarker in CT imaging has been developed reliably as an inexpensive way for classification tumors. In recent work, CD24 antibody as a biomarker for detection and characterization of cell cancer was conjugated to the Au-NPs PEGylated and used as a targeted contrast media for the detection of breast cancer 4T1 cells under in vitro and in vivo conditions [44]. In the mentioned study, they showed that AuNPs-PEGylated as CT contrast media can detect breast cancer (4T1) cells and is more effective compared with the other imaging methods. In 2017, Tang et al. [45] developed novel polyethylene glycol (PEG)-functionalized $\mathrm{Au}$ nanoparticles with star shape (AuNS@PEG) for applications in CT imaging. They have reported that the formed AuNS@PEG nanoparticles showed good bright imaging. CT images of a rat before 
injection and at different time points after intravenous injection of AuNS@PEG nanoparticles was shown in Figure 2.

Multifunctional nano-biomaterials with the integration of diagnostic and therapeutic functions have shown great capability in improving the efficacy of cancer therapy. These nanoparticles hold the potential to revolutionize future disease management. In this regard, Nuo Yu et al. [46] introduced uniform $\mathrm{Bi}$ nanoparticles capped with thiol ligands (Bi-SR) with PEGylated phospholipids (Bi-SR-PEG) as a new theranostic nanoparticle. They indicated that Bi-SR-PEG had strong near-infrared (NIR) absorbance and high photothermal conversion efficiency in addition to high X-ray sensitization and attenuation effect which can enable simultaneous tumor CT imaging and thermoradiotherapy. Inspired by the excellent performance of $\mathrm{Bi}_{2} \mathrm{~S}_{3}$ nanomaterials in photothermal therapy (PTT), a simple but powerful Bi-NPs were developed and evaluated as for PTT and MRI/PAI/CT triple-modal imaging by $\mathrm{Wu}$ et al. [47]. They have shown, that water-soluble GdPEG-Bi NPs do not only have ultrahigh X-ray attenuation coefficient but also with photoacoustic imaging (PAI) contrast effect and potent remarkable efficiency in converting NIR energy into thermal energy. In addition, Gd-PEG-Bi NPs also acted as excellent MRI contrast agents by coating gadolinium DTPA-bis-tetradecylamide (Gd-DTPA-BC16). In another work Wang et al. [48] conjugated $\mathrm{Cu}_{3} \mathrm{BiS}_{3}$ nanoparticles with target ligand folic acid (FA) which can specifically target FAreceptor overexpressed tumour cells and photosensitizer chlorin e6 (Ce6) which can further form chelate complex with $\mathrm{Gd}^{3+}$. They have found that the synthesized $\mathrm{Cu}_{3} \mathrm{BiS}_{3}-\mathrm{PEG}-$ $\left(\mathrm{Ce} 6-\mathrm{Gd}^{3+}\right)$-FA had effective dual-modal CT/MR imaging capability and excellent in vivo anti-tumour effects achieved by synergistic photothermal/photodynamic therapy.

\section{Positron Emission Tomography (PET)}

Since PET has high sensitivity, limitless depth of penetration, and quantitative capabilities, it becomes a powerful method for cancer diagnosis and functional imaging of tumors and other abnormalities [49]. PET is an essential modality for cancer staging and also the evaluation of the response of cancer to therapy. Today, due to its lack of anatomical information to identify molecular events with accurate correlation to anatomical findings, merging with these devices with either CT or MRI is known as dual imaging modalities named PET/CT and PET/MRI [50]. PET/CT has become an important method for confirming therapeutic response, providing information for the decision to stop ineffective treatment, or starting to a more efficient treatment [51]. It is helpful in localizing tumors in cases where conventional diagnostic methods are unable to localize the cancer of unknown primary.

The most useful biomarker for cancer among a variety of radiopharmaceuticals for molecular and metabolic imaging with PET is the fluorodeoxyglucose, FDG (FDG-PET/CT). Following intravenous injection, FDG, similarly to normal
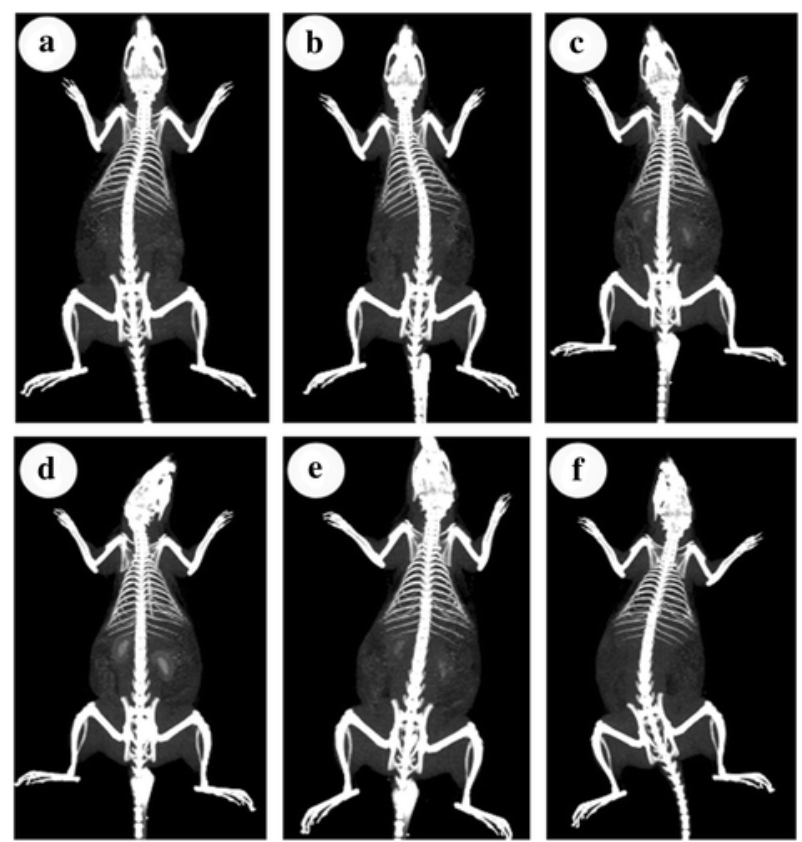

Figure 2. CT images of rat before injection (a) and at different time points $(0.5,1,2,6$, and $24 \mathrm{~h})(\mathrm{b}-\mathrm{f})$ after intravenous injection of AuNS@PEG nanoparticles (200 ppm) [45].

glucose, is taken up by cancer cells. The subsequent conversion of FDG to FDG-6-monophosphate by the intracellular enzyme hexokinase leads to trapping of the metabolite within the cancer cells [52,53]. For cancer staging, PET/CT offers many advantages over conventional imaging strategies. FDG-PET has high accuracy for staging cancers such as non-small-cell lung, gastrointestinal tract cancers including colorectal and esophageal, thyroid, head and neck, and breast [54,55].

A novel radioligand $\left({ }^{68} \mathrm{Ga}-\mathrm{NOTA}-\mathrm{AE} 105\right)$ is reported for PET which can detect the expression of uriokinase-type plasminogen activator receptors on breast, urinary bladder, and prostate cancer. This new radioligand is tested on patients diagnosed with mentioned cancers and no adverse events are reported after administration and imaging by PET/CT [56].

McDonagh et al. [57] developed an enhanced single-pot synthesis of several coated Cerium oxide nanoparticles (CONPs) and an efficient intrinsic core labeling of CONPs with the clinical PET isotope, zirconium-89, allowing detailed PET imaging and ex-vivo biodistribution. The result was demonstrated, poly (acrylic acid) coated CONPs shown excellent candidacy for clinical implementation due to their enhanced renal clearance and low reticuloendothelial system uptake. Berke et al. [58] synthesized, radiolabeled, and in vivo evaluated nanoparticles functionalized by an organosilicon fluoride acceptor ( $\mathrm{SiFA}$ ), which can be radiolabeled without further chemical modifications. The nanoparticles were radiolabeled with fluorine-18 $\left({ }^{18} \mathrm{~F}\right)$ and analyzed in vivo in a murine mammary tumor model (EMT6) in PET imaging. They have concluded that this approach represents an alternative method to produce radiolabeled nanoparticles without any further surface modification to attach a radioisotope. 
In another study, Rowe et al. evaluated the combination of MR imaging and PSMA-based PET biomarker for prostate cancer detection by utilizing imaging biomarker including ${ }^{18} \mathrm{~F}$-DCFBC PET [59].

Recently, a dual nanoprobe probe, ${ }^{99 \mathrm{~m}}$ Tc-radiolabeled nanosilica system conjugated with a trastuzumab half-chain for aggressive HER2-positive breast cancer was developed by Rainone et al. [60] as a theranostic probe. They developed 99mTc-radiolabeled nanosilica (SiNP) for targeted detection and for noninvasive detection, staging and potential treatment of HER2 positive breast cancer (HER2+ BC). Polyak et al. [61] used gallium-68 $\left({ }^{68} \mathrm{Ga}\right)$ isotope labeled porous zirconia $\left(\mathrm{ZrO}_{2}\right)$ nanoparticle (NP) with a micro-PET/CT imaging system in order to provide a nanoparticle platform which can be suitable for specific delivery of various therapeutic drugs, and suitable for PET tracing agent. They have confirmed the product's invivo radioisotope adsorbing and size stability and application as a good drug delivery agent for PET/CT imaging. Interestingly, Pascual's group conjugated mesoporous silica nanoparticle with MUC1 aptamer as a radiolabelled tracer for PET and SPECT. They have reported that ${ }^{99 \mathrm{~m}} \mathrm{Tc}(\mathrm{S} 1$-ap-MUC1Tc) showed significant targeting in tumor-bearing Balb/c mice model [62].

Norregaard et al. [63] used 2-deoxy-2-[F-18]fluoro-Dglucose $(18 \mathrm{~F}-\mathrm{FDG})$ as radiolabeled nanoparticles in PET to quantify treatment effect after photothermal therapy. They have shown that ${ }^{18} \mathrm{~F}$-FDG PET could be used as a prognostic marker for the therapeutic outcome of nanoparticle-assisted photothermal therapy in human tumor xenografts in mice.

Additionally, in SPECT several heavy radioisotopes such as ${ }^{123} \mathrm{I},{ }^{99 \mathrm{~m}} \mathrm{Tc}$, and ${ }^{133} \mathrm{Xe}$ have been applied as a radiotracers for clinical diagnosis [64]. In another study, researchers have successfully synthesized, characterized, and demonstrated the biodistribution of citrate-coated Prussian blue nanoparticles labeled using ${ }^{201} \mathrm{Tl}$ isotope in the mice. Their results have shown a biocompatible and stable ${ }^{201} \mathrm{Tl}$ labeled nanoparticulate as a SPECT tracer [65].

De Souza Albernaz et al. [66] were used an iron oxide nanoparticle conjugated with DTPA and Trastuzumab, which can efficiently be radiolabeled with ${ }^{99 \mathrm{~m}} \mathrm{Tc}$ and ${ }^{68} \mathrm{Ga}\left({ }^{99 \mathrm{~m}} \mathrm{Tc}\right.$ DTPA-TZMB@ $\mathrm{Fe}_{3} \mathrm{O}_{4}$ ) as a nanoradiopharmaceutical agent for both SPECT and PET. They have used BT-474 cells in order to induce xenografted tumor in female Balb/c nude mice. Their results were shown a clear image contrast in both SPECT and PET for breast cancer imaging.

SPECT as an excellent nuclear imaging technique, which is based on the detection of gamma-ray photons, is utilized for imaging due to its fast detection time, specificity, and affordability as compared to PET. However, SPECT is generally less sensitive than PET and also has lower spatial resolution compared to the PET, so using of micro-SPECT was recommended by researchers [67]. It was reported that the spatial resolution of micro-SPECT that is employed in the preclinical examination and animal models is higher than that of PET due to the advancement of imaging tools [67].

Moreover, SPECT/MRI has a lot of application which can provide information such as the location of the sentinel lymph node. For an instant, Misri et al. [68] conjugated iron oxide nanoparticles with ${ }^{111}$ In anti-mesothelin monoclonal antibody targeting antigens for malignant mesothelioma and suggested the dual-modality imaging probe for MRI/SPECT. Their results indicated great uptakes of superparamagnetic iron oxide nanoparticles ( ${ }^{111}$ In-MabMB-SPIONs) and signal drop after $24 \mathrm{hrs}$ post-injection.

In a new era, many researchers established the study for using multimodality probes for theranostics application. As an example, tunable and noncytotoxic PET/SPECT-MRI multimodality imaging probes using colloidally stable ligandfree SPIONs was performed by Nguyen Pham et al. [69]. In this work, they have suggested a robust and simple production for a potential next generation of nontoxic multimodality PET/SPECT-MRI single probe imaging agents.

Nowadays, in addition to the use of targeted drug specific nanocarriers in clinics, PET or SPECT allow researchers to focus on biodistribution, pharmacokinetics, and tumor targeting efficacy based on drug accumulation in the tumor site and also monitor the cancer progression.

\section{Magnetic Resonance Imaging}

The excellent features of MR imaging, as molecular imaging, include comparably high temporal and spatial resolution, excellent tissue contrast and tissue penetration, no ionizing radiation, non-invasiveness for serial studies, and simultaneous acquisition of anatomical structure and physiological function [70]. However, a limitation of MR imaging is low sensitivity which entails the introduction of an imaging agent and the development of powerful signal amplification strategies. Imaging contrast agents design is an important topic in molecular MR imaging.

MR imaging contrast agents are mainly divided into two categories: ferromagnetic and paramagnetic [71]. The former are considered as negative contrast agents which mainly reduce the signal in $\mathrm{T}_{2}$-weighted images, while the latter are referred to as positive contrast agents that increase the signal in $\mathrm{T}_{1^{-}}$ weighted images. Lauterbur et al. [72] were the first to demonstrate the feasibility of using paramagnetic contrast agents to improve tissue discrimination in MR imaging. Due to the gadolinium (Gd) labeling, the efficiency of cancer diagnosis at early stages and also drug delivery could be directly monitored by MR imaging.

In the last two decades, research efforts were concentrated on the delivery of specific $\mathrm{T}_{1}$ and $\mathrm{T}_{2}$ contrast agents into the cancer cells. The discrete contrast agents (Gd-DTPA, $\mathrm{GdCl}_{3}$, Gd-TCP, and Gd-H) and the monoclonal antibody conjugates (Gd-DTPA-9.2.27, Gd-DTPA-C595 and Gd-DTPA-WM53) 
were tested for their in vivo uptake by authors and co-workers [73-75].

In recent years, using nanoparticle and nanotechnology are interested among researchers to increase MR image contrast in particular for cancer detection in early stages. The most representative negative contrast agents are superparamagnetic iron oxide nanoparticles (SPIONs), ultrasmall superparamagnetic iron oxide nanoparticles (USPIONs), Gdencapsulated silicon micro-particles, Gd-ion doped upconversion nanoparticles (UCNPs), while typical positive contrast agents include small molecular weight compounds involving a single lanthanide chelate as signal producing element [5,76].

As mentioned earlier, the most commonly used Gd-based contrast agents for MR imaging which are among the world most recognized non-invasive techniques employed in cancer diagnosis. At ionic state, $\mathrm{Gd}$ is considered toxic but has less toxicity in its chelating form. A variety of nano-carriers, including gadolinium oxide $\left(\mathrm{Gd}_{2} \mathrm{O}_{3}\right)$ nanoparticles, have been used by researchers to improve the $T_{1}$ and $T_{2}$ contrast of MR images.

Most of the works related to the gadolinium-based layered double hydroxide and grapheme oxide were reviewed by Usman et al. in 2017 [77]. They have highlighted the use of grapheme oxide and layered double hydroxide as a suitable candidate for theranostic applications.

In a study, Shahbazi-Gahrouei et al. [78] synthesized and applied a conjugated SPIONs-C595 against ovarian cell surface for early detection of ovarian cancer. In another work, Abdolahi et al. [79] introduced SPIONs conjugated J591 Mab coupled with the extracellular domain of prostate-specific membrane antigen (PSMA) using sulfo-SMCC linker. MRI data and cell uptake experiments confirmed the high potential of the nanoprobe as a specific MRI contrast agent for the detection of PSMA-expressing prostate cancer.

In earlier work, Shahbazi-Gahrouei et al. [73] conjugated 9.2.27 and WM53 monoclonal antibodies against human melanoma and leukaemia cells, respectively, with cyclic anhydride gadolinium-dietheylenetriaminepenta-acetic (DTPA) by use of $7 \mathrm{~T}$ MRI as a useful tool for cancer diagnosis. Also, Shahbazi-Gahrouei et al. [80] introduced Gd DTPA C595 that promised contrast agents for the detection of MCF-7 breast cancer cells and Gd-hematoporphyrin for detection and diagnosis of melanoma, colorectal as well as breast cancer in MRI. In two studies [73,81] lack of using nanoparticle technology was predicted by researchers. As well as Hui Fang et al. [82] introduced Gd-DTPA-N16-16 to enhance the intensity of $\mathrm{T}_{1}$ relaxation times in the liver and kidneys of mice. They also loaded doxorubicin (DOX) into the hydrophilic cavity of vesicles and showed theranostic applications and anti-tumor effects of the DOX-loaded GdDTPA-N16-16 vesicles against MCF-7 cancer cells. Mirzaei et al. [75] introduced a dual probe, anionic linear globular dendrimer G2 with C595 Mab (Gd ${ }^{3+}$-ALGDG2-C595) as a therapeutic and imaging agent for breast cancer. Also, Shahbazi-Gahrouei et al. $[83,84]$ have conducted a study using SPIONs-C595 for detection of MUC1-expressing ovarian cancer. Their findings revealed great tumor accumulation and detection of ovarian cancer by the nontoxic nanoprobe as a specific ovarian MR imaging contrast agent.

One category of magnetic nanoparticles is magnetic iron oxide nanoparticles (MIONs) which have been notably suggested due to its great advantages. Because of the extensive application of MIONs in biomedicine, before they can be used in vivo, their cytotoxicity must be investigated [80]. In this way, Keshtkar et al. [84] prepared a novel aptamer-conjugated nanoparticle using AS1411 aptamer which was conjugated to $\mathrm{Fe}_{3} \mathrm{O}_{4} @ \mathrm{Au}$ nanoparticles on mouse mammary carcinoma (4T1) cells that overexpressed nucleolin. They have found that the synthesized nanoprobe produced strongly darkened $\mathrm{T}_{2-}$ weighted (90\% reduction of signal intensity) in 4T1 cells at $45 \mu \mathrm{g} / \mathrm{mL}$ concentration. Recently, Ghahremani et al. in 2018 $[85,86]$ introduced bovine serum albumin (BSA) capped gold nanoclusters targeted by AS1411 aptamer as a gold radiosensitizing (with a size of $10-15 \mathrm{~nm}$ ) candidate for megavoltage radiotherapy and biomedical imaging of breast cancer cells (4T1) targeting agent.

Moradi Khaniabadi et al. [4,5] conducted a study on SPIONs conjugated with C595 Mab, MR $\mathrm{T}_{2}$ contrast agent for early detection of overexpressed MUC1 breast cancer. The findings demonstrated that MR images significantly enhanced the intensity of $T_{1}$ and $T_{2}$ relaxation times of breast cancer cells. The cellular uptake of nanoprobe showed a great cellular internalization in the MCF-7 cells, in comparison with control. They also concluded that SPIONs-C595 exhibited high dual $\left(\mathrm{T}_{1}\right.$ and $\mathrm{T}_{2}$ ) $\mathrm{MR}$ imaging contrast potential and might be applied for specific breast cancer (MCF-7) cell detection.

Manganese oxide nanoparticles (MnO NPs)-based MRI contrast agents are another category of nanoprobes which has significant attention due to its ability of the diagnosis of brain disorders in $\mathrm{T}_{1}$-weighted images. A one-pot approach for the preparation of hydrophilic MnO NPs as MRI contrast agent was proposed by Li et al. [87]. They have targeted PEG-MnO NPs with amino group modified AS1411 and indicated the promising potential of PEG-MnO NPs as $\mathrm{T}_{1}$ MRI contrast agent for the molecular MR imaging of 786-0 renal carcinoma.

Hydrophilic $\mathrm{CaF}_{2}: \mathrm{Yb}, \mathrm{Er} @ \mathrm{CaF}_{2}: \mathrm{Gd}$ nanoparticles modified using polyethylene glycol-polyamidoamine (PEG-PAA) diblock copolymer benefited from the presence of Gd only in the outer $\mathrm{CaF}_{2}$ layer of the nanoparticles for efficient $\mathrm{MR}$ angiography (MRA) and cancer detection which was developed by Liu $\mathrm{K}$ et al. [88]. In another work, $\mathrm{NaErF}_{4} @ \mathrm{NaGdF}_{4}(\mathrm{Er} @ \mathrm{Gd})$ nanoparticle evaluated by Liyi Ma et al. [89] for MRI and short wave infra-red (SWIR) imaging. They have conjugated Er@Gd nanoparticle with cypate and could simultaneously provide SWIR imaging and $\mathrm{T}_{1}$-weighted MRI of tumors in mice and effective photothermal therapy to ablate tumors. In addition, You Qing et al. [90] constructed an 
innovative multifunctional drug delivery platform by (Gd)based BSA hybrid-coated hollow gold nanoshells (Au@BSAGd). Innovative nanoprobe, Au@BSA-Gd, was applied for near-infrared fluorescence and excellent $T_{1}$ MR imaging contrast agent for capability as indicated in Figure 3 [90].

A multimodal contrast agent for integrated preoperative MR imaging and intra-operative fluorescence image-guided surgery (FIGS) was used by Payne et al. [91[. They have employed simulated surgical phantoms that were routinely used to evaluate the depth at which NIR imaging agents could be detected by FIGS.

The quantum dots (QD) probes based on semiconductor quantum dots have been introduced by researchers, and its applications in biological systems was published in Nature magazine which, marked era arrival of fluorescence QD labeling biological molecules [92,93]. QD probes can specially label biomarkers on tumor cell surface and accurately label subtle sub-cellular structure. Application of nanoprobes with MR imaging-Fluorescence imaging (MRI-FI) as dualmodality at the same time have displayed the spatial distribution of breast cancer stem cells (BCSCs) in solid tumors [93].

Recently, ${ }^{19} \mathrm{~F}$ MRI has attracted significant attention due to several unique advantages such as low background signals, high signal-to-noise ratio (SNR), $100 \%$ natural isotopic abundance, relatively high sensitivity comparable to that of ${ }^{1} \mathrm{H}$ and the wide range of chemical shifts [94]. In 2018, Minmin $\mathrm{Xu}$ et al. [95] presented a new strategy to integrate the complementary modalities of ${ }^{19} \mathrm{~F}$ MRI and fluorescence imaging (FI) into a polymer nanoprobe. Their synthesized nanoprobe composed of hydrophobic fluorescent organic core, azide modified tetra-phenylethylene (TPE-N3) and hydrophilic fluorinated polymer shell which could successfully enter into the HeLa cells, and emit an obvious blue fluorescence originated from the TPE core and enhanced MR signals.

\section{Optical Imaging}

Optical imaging is a non-invasive modality for looking inside the body, which uses visible light and the special properties of photons, infrared, and ultraviolet to obtain detailed images of organs and small structures like cells and molecules. Furthermore, combination optical imaging with other modalities like MR imaging, CT or X-ray, could be enhanced the information from living body organs. This new molecular imaging modality includes a variety of techniques such as Endoscopy, Optical Coherence Tomography (OCT), Photoacoustic Imaging (PAI), Raman Spectroscopy, Diffuse Optical Tomography (DOT), and Super-resolution Microscopy.

Recently, several studies have focused on the application of nanoparticles in optical imaging. Fabrication and characterization of silica coated $\mathrm{PbS}$ quantum as optical nanoprobe to target the $\alpha v \beta 3$ integrin receptor on an angiogenic tumor in NIR region have performed by Depalo et al. [96].

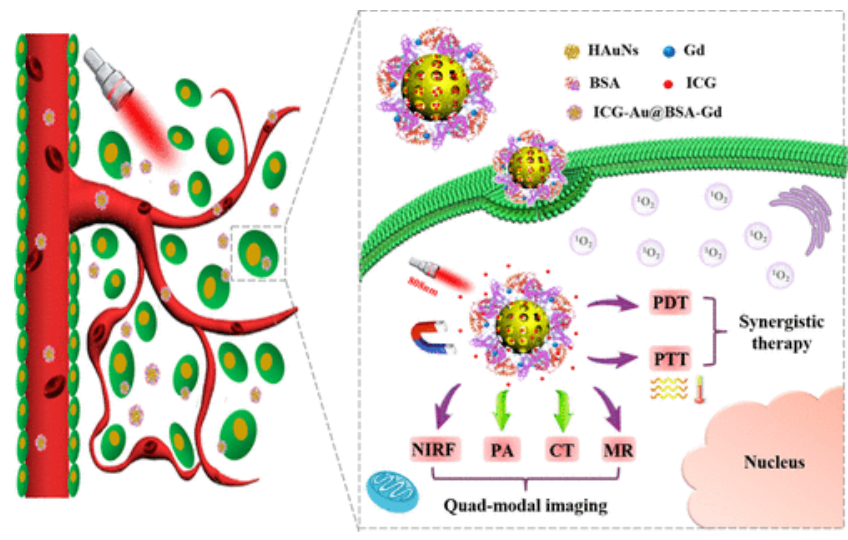

Figure 3. Schematic diagram for multimodal imaging, using ICGAu@BSA-Gd $T_{1}$ contrast media for cancer imaging and therapy [90].

In another work, a novel nuclear targeting nanoprobe, Au NPs conjugated to SV-4 was presented by Xie et al. [97]. They have shown this probe could assess the cell nucleus and provide significant information in living cells which employed (surface-enhanced Raman scattering) SERS for the research of drug delivery and cancer therapy. In addition, the applicability of melanin coated gold nanorods as a photoacoustic contrast agent was evaluated by Repenko et al. [98]. They have indicated photoacoustic contrast produced by nanoprobe in mouse intestine greatly improved over the pure melanin or pristine gold particles.

In another study, novel porous gold nanocluster decorated manganese monoxide nanocomposites ( $\mathrm{MnO} @ \mathrm{Au} \mathrm{NCs}$ ) were synthesized by $\mathrm{Yu} \mathrm{Liu} \mathrm{et} \mathrm{al.} \mathrm{[99]} \mathrm{and} \mathrm{used} \mathrm{as} \mathrm{MRI/PAI/CT}$ tumor tri-modal imaging. The broad NIR absorption of $\mathrm{MnO} @ \mathrm{Au}$ NCs significantly enhanced the PA imaging depth and porous architecture of it, enhanced the accessibility of the $\mathrm{Mn}$ centers to proximal water molecules and improved $\mathrm{T}_{1^{-}}$ weighted MR imaging of tumor. Meanwhile, the $\mathrm{Au}$ nanoclusters could serve as CT imaging which helped delayed $\mathrm{T}_{1}$ contrast and deeper PAI depth.

Nowadays, scientists put much effort to develop and synthesize new versatile nanoprobes to provide prominent information on the tumor by improving contrast for imaging. A new multifunctional nanoplatform for amplified phototherapy by concurrently achieving mitochondria-targeting phototherapy, synergistic photothermal therapy (PTT)/photodynamic therapy (FDT) by a single NIR irradiation (808 nm), self-sufficient oxygen-augmented, and multipleimaging guidance/monitoring was designed by Liang Zhang et al [100]. They prepared a "Nano-RBCs (red blood cells)" nanosystem by fabricating perfluorooctyl bromide (PFOB)based nanoliposomes and integrated IR780 for concurrent and synergistic PTT and PDT. PFOB@LIP-IR780 "Nano-RBCs" showed the high oxygen delivery property for amplified PDT and mitochondria-targeting performance for enhanced and synergistic PDT/PTT. Furthermore, based on the unique bromine atom in $\mathrm{PFOB}$, excellent photoluminescence property 
of IR780 and desirable photothermal-conversion performance of the nanocarrier, PFOB @LIP-IR780 "Nano-RBCs" could act as the contrast agents for concurrent CT, PA, and FL multiple imaging.

The application of NIR-emitting quantum dots in cancer diagnostic and detection is an important issue in modern nanobiotechnology. Recently, the importance of NIR imaging has dramatically increased due to the lowest absorption in tissue and the deepest tissue imaging and the availability of targeted biological agents for diagnosis [101] In this way, Ren and co-workers [102] prepared monodisperse Neodymium (Nd3)-doped down-conversion nanocrystals for NIR/CT/MRI imaging of breast tumor which was established by the subcutaneous inoculation of 4T1 cells. They have reported that among the resultant core-shell nanocrystals, $\mathrm{NaYF}_{4}: 5 \% \mathrm{Nd} @ \mathrm{NaGdF}_{4}$ exhibit the brightest luminescence in the NIR II window, strong temperature-dependent paramagnetism and fast attenuation towards X-rays. Pisanic et al. [103] reported overview literature of quantum dots and paradigm examples of how these phenomena have been readily exploited for manifold uses in diagnostic and cancer detection.

Synthesis of luminescent carbon dots (CDs) with ultrahigh quantum yield and inherent folate receptor-positive cancer cell targetability was performed by Liu $\mathrm{H}$ et al. [104]. They showed excellent photoluminescent activity, high photostability, and favorable biocompatibility of CDs and its great potential in biological and bioimaging studies.

Persistent luminescence nanoparticles offer attractive capabilities for in vivo imaging applications as an optical excitation of the nanoparticles outside the animal before the injection is possible. Pellerin et al. [105] developed the Lanthanum Aluminum Oxide $\left(\mathrm{LaAlO}_{3}\right)$ nano-perovskite doped with Chromium $\left(\mathrm{Cr}^{3+}\right)$ and Samarium $\left(\mathrm{Sm}^{3+}\right)$ to enhance the persistent properties. Synthesized NPs presented deep red longlasting persistent luminescence at $734 \mathrm{~nm}$ after UV excitation allowed performing in vivo imaging with high signal to noise ratio and with high sensitivity.

New application and comparison of graphene quantum dots (GQDs) were considered by Schroeder et al. [106]. They compared and discussed the ability of state-of-the-art use of GQDs in biology and health sciences. In Hai and co-workers' study, the conjugation of terephthalic acid (TPA) on the surface of graphene quantum dots (TPA@GQDs) was demonstrated by the quantitative fluorescent imaging of hydroxyl radical in living HeLa cells under different circumstances [107]. They showed its low cytotoxicity and favorable biocompatibility and also recommended it as a potential image modality for cancer diagnosis.

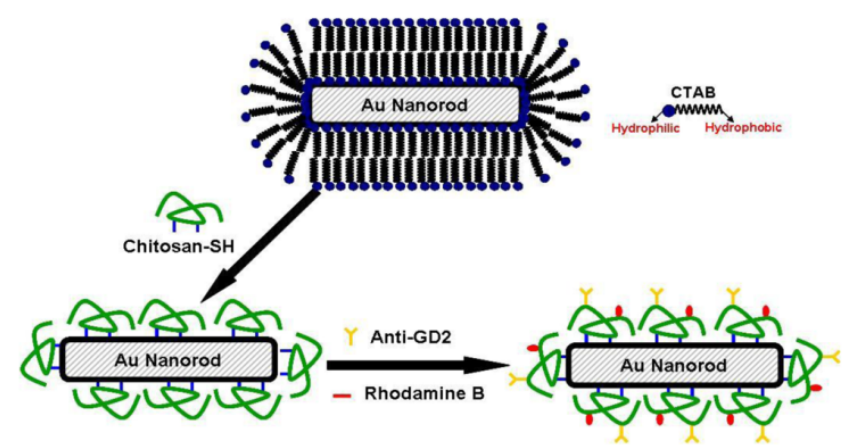

Figure 4. The nanostructure and shape of Gold nanorods (AuNRs).

\section{Discussion}

As stated, different molecular imaging modalities such as PET, SPECT, MRI, CT, Ultrasound and Optical imaging using a variety of nanoprobes are widely used for cancer diagnosis. In this review, a broad overview of nanoparticles applications in molecular imaging modalities was provided for cancer detection. Overall, as highlighted in this article, MRI and PET are more applicable compared to the other modalities for cancer diagnosis. Among all mentioned nanoprobes, gold nanoparticles (Au-NPs), have been applied and had a unique role for use in drug delivery, targeting therapy and molecular imaging. The biologically relevant size, low inherent toxicity, high surface area, and ability to easily functionalize with biomolecules, as well as their enhanced optical properties provides it with unique attributes that help to improve therapeutic delivery, imaging, and noninvasive disease diagnostics. The nanostructure and shape of the nanostructures of gold nanorods (Au-NRs) is presented in Figure 4.

\section{Conclusion}

Multimodality imaging is of great importance in diagnostic of cancer at early stages. This review article could be useful as a tutorial and educational view for all medical students and nanotechnology researchers who are interested in cancer imaging and therapy. The limitation of this article is that have not covered every aspect of all imaging modalities for cancer detection in details. With knowledge of the correlation between the application of nanoparticles and molecular imaging modalities and with the development of targeted contrast agents or nanoprobes, they may provide better cancer diagnosis in the future. The outcome of this study may help the design of tumor-specific contrast agents.

\section{Acknowledgment}

This work is a part of projects (No: 194116 and 196053) which was financially supported by the Isfahan University of Medical Sciences, Isfahan, Iran. 


\section{References}

[1] Hussain T, Nguyen QT. Molecular imaging for cancer diagnosis and surgery. Advanced Drug Delivery Reviews. 2014;66:90-100.

[2] Sun X, Li Y, Liu T, et al. Peptide-based imaging agents for cancer detection. Advanced Drug Delivery Reviews. 2017;110:38-51.

[3] Kircher MF, Hricak H, Larson SM. Molecular imaging for personalized cancer care. Molecular Oncology. 2012;6(2):182-195.

[4] Khaniabadi PM, Shahbazi-Gahrouei D, Jaafar MS, et al. Magnetic iron oxide nanoparticles as T2 MR imaging contrast agent for detection of breast cancer (MCF-7) cell. Avicenna Journal of Medical Biotechnology. 2017;9(4):181-188.

[5] Khaniabadi PM, Majid A, Asif M. et al. Breast cancer cell targeted MR molecular imaging probe: Anti-MUC1 antibody-based magnetic nanoparticles. Journal of Physics: Conference Series. 2017;851(1):012014.

[6] Mirzaei M, Mohagheghi M, Shahbazi-Gahrouei D. Synthesis and development of Gd3+-ALGDG2-C595 as MR Imaging Contrast Agent. Journal of Biomaterials and Nanobiotechnology. 2013;4(01):22.

[7] Khemthongcharoen N, Jolivot R, Rattanavarin S, Piyawattanametha W. Advances in imaging probes and optical microendoscopic imaging techniques for early in vivo cancer assessment. Advanced Drug Delivery Reviews. 2014;74:53-74.

[8] Savla R, Minko T. Nanoparticle design considerations for molecular imaging of apoptosis: Diagnostic, prognostic, and therapeutic value. Advanced Drug Delivery Reviews. 2017;113:122-140.

[9] Muthu MS, Leong DT, Mei L, Feng S-S. Nanotheranostics- application and further development of nanomedicine strategies for advanced theranostics. Theranostics. 2014;4(6):660-677.

[10] Guo R, Lu G, Qin B, Fei B. Ultrasound Imaging Technologies for Breast Cancer Detection and Management: A Review. Ultrasound in Medicine and Biology. 2018;44(1):37-70.

[11] Li N, Han L, Jing H. Contrast-enhanced ultrasound with a novel nanoparticle contrast agent for clinical diagnosis in patients with non-small cell lung cancer. Experimental and Therapeutic Medicine. 2017;14(4):3768-3773.

[12] Paefgen V, Doleschel D, Kiessling F. Evolution of contrast agents for ultrasound imaging and ultrasound-mediated drug delivery. Frontiers in Pharmacology. 2015;6:197.

[13] Beik J, Shiran MB, Abed Z, et al. Gold nanoparticle-induced sonosensitization enhances the antitumor activity of ultrasound in colon tumor-bearing mice. Medical Physics. 2018;45(9):4306-4314.

[14] Milgroom A, Intrator M, Madhavan K, et al. Mesoporous silica nanoparticles as a breast-cancer targeting ultrasound contrast agent. Colloids and Surfaces B, Biointerfaces. 2014;116:652-657.

[15] Martins SF, Garcia EA, Luz MA, et al. Clinicopathological correlation and prognostic significance of VEGF-A, VEGF-C, VEGFR-2 and VEGFR-3 expression in colorectal cancer. Cancer Genomics \& Proteomics. 2013;10(2):55-67.

[16] Abou-Elkacem L, Wilson KE, Johnson SM, et al. Ultrasound Molecular Imaging of the Breast Cancer Neovasculature using Engineered Fibronectin Scaffold Ligands: A Novel Class of Targeted Contrast Ultrasound Agent. Theranostics. 2016;6(11):17401752.

[17] Hao L, Wang Z, Luo J, et al. Preparation of targeted high molecular polymer microbubble encapsuling quantum dots and cell experiment study in vitro. Journal of Chongqing Medical University. 2013(1):24-27.

[18] Lutz AM, Bachawal SV, Drescher CW, et al. Ultrasound molecular imaging in a human CD276 expression-modulated murine ovarian cancer model. Clinical Cancer Research. 2014;20(5):1313-1322.

[19] Yoon YI, Pang X, Jung S, et al. Smart gold nanoparticle-stabilized ultrasound microbubbles as cancer theranostics. Journal of Materials Chemistry B. 2018;6(20):3235-3239.

[20] Hernot S, Klibanov AL. Microbubbles in ultrasound-triggered drug and gene delivery. Adv Drug Deliv Rev. 2008;60(10):1153-1166.

[21] Yildirim A, Shi D, Roy S, et al. Nanoparticle-Mediated Acoustic Cavitation Enables High Intensity Focused Ultrasound Ablation Without Tissue Heating. ACS Applied Materials \& Interfaces. 2018;10(43):36786-36795.

[22] McLaughlan J, Cowell D, Freear S. Gold nanoparticle nucleated cavitation for enhanced high intensity focused ultrasound therapy. Physics in Medicine \& Biology. 2017;63(1):015004.

[23] Nedaei TS, Delavari HH. Preparation of Naturally Active Melanin Nano-Platforms Chelated with Barium Ions as a Potential X-Ray-Computed Tomography Contrast Agent. ChemistrySelect. 2018;3(39):11098-11102.

[24] Wallyn J, Anton N, Serra CA, et al. A new formulation of poly (MAOTIB) nanoparticles as an efficient contrast agent for in vivo Xray imaging. Acta Biomaterialia. 2018;66:200-212.

[25] Hainfeld JF, Ridwan SM, Stanishevskiy Y, et al. Small, Long Blood Half-Life Iodine Nanoparticle for Vascular and Tumor Imaging. Scientific Reports. 2018;8(1):13803.

[26] Goldman LW. Principles of CT and CT technology. J Nucl Med Technol. 2007;35(3):115-28; quiz 29-30.

[27] Kim D, Yu MK, Lee TS, et al. Amphiphilic polymer-coated hybrid nanoparticles as CT/MRI dual contrast agents. Nanotechnology. 2011;22(15):155101.

[28] Gu X, Liu R. Application of 18F-FDG PET/CT combined with carbohydrate antigen 19-9 for differentiating pancreatic carcinoma from chronic mass-forming pancreatitis in Chinese elderly. Clin Interv Aging. 2016;11:1365-1370.

[29] Zheng X, Wang S, Wu L, Hou X. Microwave-assisted facile synthesis of mono-dispersed Ba/Ho co-doped nanohydroxyapatite for potential application as binary CT imaging contrast agent. Microchemical Journal. 2018;141:330-336.

[30] Naha PC, Lau KC, Hsu JC, et al. Gold silver alloy nanoparticles (GSAN): an imaging probe for breast cancer screening with dualenergy mammography or computed tomography. Nanoscale. 2016;8(28):13740-13754.

[31] Shi X, Wang SH, Van Antwerp ME, et al. Targeting and detecting cancer cells using spontaneously formed multifunctional dendrimer-stabilized gold nanoparticles. The Analyst. 2009;134(7):1373-1379. 
[32] Eck W, Nicholson AI, Zentgraf H, et al. Anti-CD4-targeted gold nanoparticles induce specific contrast enhancement of peripheral lymph nodes in X-ray computed tomography of live mice. Nano Letters. 2010;10(7):2318-2322.

[33] Zhang Z, Ross RD, Roeder RK. Preparation of functionalized gold nanoparticles as a targeted X-ray contrast agent for damaged bone tissue. Nanoscale. 2010;2(4):582-586.

[34] Aydogan B, Li J, Rajh T, et al. AuNP-DG: Deoxyglucose-labeled gold nanoparticles as X-ray computed tomography contrast agents for cancer imaging. Molecular Imaging and Biology. 2010;12(5):463-467.

[35] Zhou B, Yang J, Peng C, et al. PEGylated polyethylenimine-entrapped gold nanoparticles modified with folic acid for targeted tumor CT imaging. Colloids and surfaces B, Biointerfaces. 2016;140:489-496.

[36] Khademi S, Sarkar S, Shakeri-Zadeh A, et al. Folic acid-cysteamine modified gold nanoparticle as a nanoprobe for targeted computed tomography imaging of cancer cells. Materials Science and Engineering: C. 2018;89:182-193.

[37] Zhang Y, Wen S, Zhao L, et al. Ultrastable polyethyleneimine-stabilized gold nanoparticles modified with polyethylene glycol for blood pool, lymph node and tumor CT imaging. Nanoscale. 2016;8(10):5567-5577.

[38] Nakagawa T, Gonda K, Kamei T, et al. X-ray computed tomography imaging of a tumor with high sensitivity using gold nanoparticles conjugated to a cancer-specific antibody via polyethylene glycol chains on their surface. Science and Technology of Advanced Materials. 2016;17(1):387-297.

[39] Kobayashi Y, Shibuya K, Tokunaga M, et al. Preparation of high-concentration colloidal solution of silica-coated gold nanoparticles and their application to X-ray imaging. Journal of Sol-Gel Science and Technology. 2016;78(1):82-90.

[40] Cole LE, Ross RD, Tilley JM, et al. Gold nanoparticles as contrast agents in X-ray imaging and computed tomography. Nanomedicine. 2015;10(2):321-341.

[41] Jing L, Liang X, Deng Z, et al. Prussian blue coated gold nanoparticles for simultaneous photoacoustic/CT bimodal imaging and photothermal ablation of cancer. Biomaterials. 2014;35(22):5814-5821.

[42] Zhang J, Li C, Zhang X, et al. In vivo tumor-targeted dual-modal fluorescence/CT imaging using a nanoprobe co-loaded with an aggregation-induced emission dye and gold nanoparticles. Biomaterials. 2015;42:103-111.

[43] Bi H, He F, Dong Y, et al. Bismuth Nanoparticles with "Light" Property Served as a Multifunctional Probe for X-ray Computed Tomography and Fluorescence Imaging. Chemistry of Materials. 2018;30(10):3301-3307.

[44] Fazel-Ghaziyani M, Shahbazi-Gahrouei D, Pourhassan-Moghaddam M, et al. Targeted detection of the cancer cells using the antiCD24 bio modified PEGylated gold nanoparticles: the application of CD24 as a vital cancer biomarker. Nanomedicine J. 2018;5(3):172-179.

[45] Tang D, Gao W, Yuan Y, et al. Novel Biocompatible Au Nanostars@ PEG Nanoparticles for In Vivo CT Imaging and Renal Clearance Properties. Nanoscale Research Letters. 2017;12(1):565.

[46] Yu N, Wang Z, Zhang J, et al. Thiol-capped Bi nanoparticles as stable and all-in-one type theranostic nanoagents for tumor imaging and thermoradiotherapy. Biomaterials. 2018;161:279-291.

[47] $\mathrm{Wu} \mathrm{B}$, Lu S-T, Yu H, et al. Gadolinium-chelate functionalized bismuth nanotheranostic agent for in vivo MRI/CT/PAI imagingguided photothermal cancer therapy. Biomaterials. 2018;159:37-47.

[48] Wang $\mathrm{Y}$, Cai $\mathrm{D}$, $\mathrm{Wu} \mathrm{H}$, et al. Functionalized $\mathrm{Cu} 3 \mathrm{BiS} 3$ nanoparticles for dual-modal imaging and targeted photothermal/photodynamic therapy. Nanoscale. 2018;10(9):4452-1162.

[49] Kaufmann PA, Di Carli MF, editors. Hybrid SPECT/CT and PET/CT imaging: the next step in noninvasive cardiac imaging. Seminars in nuclear medicine; 2009: Elsevier.

[50] Antoch G, Bockisch A. Combined PET/MRI: a new dimension in whole-body oncology imaging? European Journal of Nuclear Medicine and Molecular Imaging. 2009;36(Suppl1):113-120.

[51] Giovacchini G, Picchio M, Garcia-Parra R, et al. 11C-choline PET/CT predicts prostate cancer-specific survival in patients with biochemical failure during androgen-deprivation therapy. Journal of Nuclear Medicine. 2014;55(2):233-241.

[52] Takenaka D, Ohno Y, Koyama H, et al. Integrated FDG-PET/CT vs. standard radiological examinations: comparison of capability for assessment of postoperative recurrence in non-small cell lung cancer patients. European Journal of Radiology. 2010;74(3):458464.

[53] Buck AK, Herrmann K, Stargardt T, et al. Economic evaluation of PET and PET/CT in oncology: evidence and methodologic approaches. Journal of Nuclear Medicine Technology. 2010;38(1):6-17.

[54] Miyazaki T, Sohda M, Higuchi T, et al. Effectiveness of FDG-PET in screening of synchronous cancer of other organs in patients with esophageal cancer. Anticancer Research. 2014;34(1):283-287.

[55] Khan N, Oriuchi N, Higuchi T, et al. PET in the follow-up of differentiated thyroid cancer. British Journal of Radiology. 2003;76(910):690-695.

[56] Skovgaard D, Persson M, Brandt-Larsen M, et al. Safety, dosimetry, and tumor detection ability of 68Ga-NOTA-AE105: First-inhuman study of a novel radioligand for uPAR PET imaging. Journal of Nuclear Medicine. 2017;58(3):379-386.

[57] McDonagh PR, Sundaresan G, Yang L, et al. Biodistribution and PET imaging of 89-zirconium labeled cerium oxide nanoparticles synthesized with several surface coatings. Nanomedicine: Nanotechnology, Biology and Medicine. 2018;14(4):1429-1440.

[58] Berke S, Kampmann A-L, Wuest M, et al. 18F-Radiolabeling and In Vivo Analysis of SiFA-Derivatized Polymeric Core-Shell Nanoparticles. Bioconjugate Chemistry. 2017;29(1):89-95.

[59] Rowe SP, Gage KL, Faraj SF, et al. 18F-DCFBC PET/CT for PSMA-based detection and characterization of primary prostate cancer. Journal of Nuclear Medicine. 2015;56(7):1003-1010. 
[60] Rainone P, Riva B, Belloli S, et al. Development of 99mTc-radiolabeled nanosilica for targeted detection of her2-positive breast cancer. International Journal of Nanomedicine. 2017;12:3447-3461.

[61] Polyak A, Nagy LN, Mihaly J, et al. Preparation and 68Ga-radiolabeling of porous zirconia nanoparticle platform for PET/CTimaging guided drug delivery. Journal of Pharmaceutical and Biomedical Analysis. 2017;137:146-150.

[62] Pascual L, Cerqueira-Coutinho C, García-Fernández A, et al. MUC1 aptamer-capped mesoporous silica nanoparticles for controlled drug delivery and radio-imaging applications. Nanomedicine: Nanotechnology, Biology and Medicine. 2017;13(8):2495-2505.

[63] Norregaard K, Jørgensen JT, Simón M, et al. 18F-FDG PET/CT-based early treatment response evaluation of nanoparticle-assisted photothermal cancer therapy. PloS one. 2017;12(5):e0177997.

[64] Cassidy PJ, Radda GK. Molecular imaging perspectives. Journal of the Royal Society Interface. 2005;2(3):133-144.

[65] Szigeti K, Hegedüs N, Rácz K, et al. Thallium Labeled Citrate-Coated Prussian Blue Nanoparticles as Potential Imaging Agent. Contrast Media and Molecular Imaging. 2018;2018:2023604.

[66] de Souza Albernaz M, Toma SH, Clanton J, et al. Decorated Superparamagnetic Iron Oxide Nanoparticles with Monoclonal Antibody and Diethylene-Triamine-Pentaacetic Acid Labeled with Thechnetium-99m and Galium-68 for Breast Cancer Imaging. Pharmaceutical Research. 2018;35(1):24.

[67] Thomas R, Park I-K, Jeong Y. Magnetic iron oxide nanoparticles for multimodal imaging and therapy of cancer. International Journal of Molecular Sciences. 2013;14(8):15910-15930.

[68] Misri R, Meier D, Yung AC, et al. Development and evaluation of a dual-modality (MRI/SPECT) molecular imaging bioprobe. Nanomedicine: Nanotechnology, Biology and Medicine. 2012;8(6):1007-1016.

[69] Pham TN, Lengkeek NA, Greguric I, et al. Tunable and noncytotoxic PET/SPECT-MRI multimodality imaging probes using colloidally stable ligand-free superparamagnetic iron oxide nanoparticles. International Journal of Nanomedicine. 2017;12:899-909.

[70] Bennett KM, Jo J, Cabral H, et al. MR imaging techniques for nano-pathophysiology and theranostics. Advanced Drug Delivery Reviews. 2014;74:75-94.

[71] Shahbazi-Gahrouei D. Novel MR imaging contrast agents for cancer detection. Journal of Research in Medical Sciences. 2009;14(3):141-147.

[72] Lauterbur P. Image-formation by induced local interactions-examples employing nuclear magnetic-resonance. Clinical Orthopaedics and Related Research. 1989;244:3-6.

[73] Shahbazi-Gahrouei D, Williams M, Rizvi S, Allen B. In vivo studies of Gd-DTPA-monoclonal antibody and gd-porphyrins: Potential magnetic resonance imaging contrast agents for melanoma. Journal of Magnetic Resonance Imaging. 2001;14(2):169-174.

[74] Shahbazi-Gahrouei D, Rizvi S, Williams M, Allen B. In vitro studies of gadolinium-DTPA conjugated with monoclonal antibodies as cancer-specific magnetic resonance imaging contrast agents. Australasian Physics and Engineering Sciences in Medicine. 2002;25(1):31-38.

[75] Mirzaei M, Mohagheghi M, Shahbazi-Gahrouei D, Khatami A. Gd3+-Anionic Linear Globular Dendrimer-G2-C595 A Dual Novel Nanoprobe for MR Imaging and Therapeutic Agent. J Nanomed Nanotechnol. 2012;3(7):1000147.

[76] Zhang M, Cao Y, Chong Y, et al. Graphene oxide based theranostic platform for T 1-weighted magnetic resonance imaging and drug delivery. ACS Applied Materials and Interfaces. 2013;5(24):13325-13332.

[77] Shahbazi-Gahrouei D, Abdolahi M. A novel method for quantitative analysis of anti-MUC1 expressing ovarian cancer cell surface based on magnetic cell separation. Journal of Medical Sciences. 2012;12(8):256-266.

[78] Abdolahi M, Shahbazi-Gahrouei D, Laurent S, et al. Synthesis and in vitro evaluation of MR molecular imaging probes using J591 mAb-conjugated SPIONs for specific detection of prostate cancer. Contrast Media and Molecular Imaging. 2013;8(2):175-184.

[79] Shahbazi-Gahrouei D, Abdolahi M. Detection of MUC1-expressing ovarian cancer by C595 monoclonal antibody-conjugated SPIONs using MR imaging. The Scientific World Journal. 2013;2013:609151.

[80] Shahbazi-Gahrouei D, Abdolahi M. Superparamagnetic iron oxide-C595: Potential MR imaging contrast agents for ovarian cancer detection. Journal of Medical Physics. 2013;38(4):198-204.

[81] Shahbazi-Gahrouei D, Khodamoradi E. Porphyrin-based agents: potential MR imaging contrast agents for colorectal (HT29/219) detection in mice. Journal of Medical Sciences. 2007;7(6):1015-1020.

[82] Fang H, Liu C, Liu C, et al. Gd-DTPA-dialkylamine derivatives: Synthesis and self-assembled behaviors for T1-enhanced magnetic resonance imaging and drug carriers. Journal of Molecular Liquids. 2018;268:77-86.

[83] Keshtkar M, Shahbazi-Gahrouei D, Mehrgardi M, Aghaei M. Synthesis and Cytotoxicity Assessment of Gold-coated Magnetic Iron Oxide Nanoparticles. Journal of Biomedical Physics and Engineering. 2018;8(4):357-364.

[84] Keshtkar M, Shahbazi-Gahrouei D, Khoshfetrat SM, et al. Aptamer-conjugated magnetic nanoparticles as targeted magnetic resonance imaging contrast agent for breast cancer. Journal of Medical Signals and Sensors. 2016;6(4):243-247.

[85] Ghahremani F, Shahbazi-Gahrouei D, Kefayat A, et al. AS1411 aptamer conjugated gold nanoclusters as a targeted radiosensitizer for megavoltage radiation therapy of 4T1 breast cancer cells. RSC Advances. 2018;8(8):4249-4258.

[86] Ghahremani F, Kefayat A, Shahbazi-Gahrouei D, Motaghi H, Mehrgardi MA, Javanmard SH. AS1411 Aptamer targeted gold nanoclusters effect on enhancement of radiation therapy efficacy in 4T1 breast tumor-bearing mice. Nanomedicine. 2018; 13(20): 2563-2578.

[87] $\mathrm{Li} \mathrm{J}, \mathrm{Wu} \mathrm{C}$, Hou P, et al. One-pot preparation of hydrophilic manganese oxide nanoparticles as T1 nano-contrast agent for molecular magnetic resonance imaging of renal carcinoma in vitro and in vivo. Biosensors and Bioelectronics. 2018;102:1-8.

[88] Liu K, Yan X, Xu Y-J, et al. Sequential growth of CaF 2: Yb, Er@ CaF 2: Gd nanoparticles for efficient magnetic resonance angiography and tumor diagnosis. Biomaterials Science. 2017;5(12):2403-2415. 
[89] Ma L, Liu Y, Liu Liu L, et al. Simultaneous activation of short-wave infrared (SWIR) light and paramagnetism by a functionalized shell for high penetration and spatial resolution theranostics. Advanced Functional Materials. 2018;28(6):1705057.

[90] You Q, Sun Q, Yu M, et al. BSA-Bioinspired Gadolinium Hybrid-Functionalized Hollow Gold Nanoshells for NIRF/PA/CT/MR Quadmodal Diagnostic Imaging-Guided Photothermal/Photodynamic Cancer Therapy. ACS Applied Materials \& Interfaces. 2017;9(46):40017-40030.

[91] Payne WM, Hill TK, Svechkarev D, et al. Multimodal imaging nanoparticles derived from hyaluronic acid for integrated preoperative and intraoperative cancer imaging. Contrast Media and Molecular Imaging. 2017;2017:9616791.

[92] Chan WC, Nie S. Quantum dot bioconjugates for ultrasensitive nonisotopic detection. Science. 1998;281(5385):2016-2018.

[93] Chen H, Wang Y, Wang T, et al. Application prospective of nanoprobes with MRI and FI dual-modality imaging on breast cancer stem cells in tumor. Journal of Nanobiotechnology. 2016;14(1):52.

[94] Srinivas M, Heerschap A, Ahrens ET, et al. 19F MRI for quantitative in vivo cell tracking. Trends in Biotechnology. 2010;28(7):363370.

[95] Xu M, Guo C, Hu G, et al. Organic Nanoprobes for Fluorescence and 19F Magnetic Resonance Dual-Modality Imaging. Chinese Journal of Chemistry. 2018;36(1):25-30.

[96] Depalo N, Corricelli M, De Paola I, et al. NIR Emitting Nanoprobes Based on Cyclic RGD Motif Conjugated PbS Quantum Dots for Integrin-Targeted Optical Bioimaging. ACS Appl Mater Interfaces. 2017;9(49):43113-43126.

[97] Xie W, Wang L, Zhang Y, et al. Nuclear targeted nanoprobe for single living cell detection by surface-enhanced Raman scattering. Bioconjug Chem. 2009;20(4):768-773.

[98] Repenko T, Rix A, Nedilko A, et al. Strong photoacoustic signal enhancement by coating gold nanoparticles with melanin for biomedical imaging. Advanced Functional Materials. 2018;28(7):1705607.

[99] Liu Y, Lv X, Liu H, et al. Porous gold nanocluster-decorated manganese monoxide nanocomposites for microenvironmentactivatable MR/photoacoustic/CT tumor imaging. Nanoscale. 2018;10(8):3631-3638.

[100] Zhang L, Wang D, Yang K, et al. Mitochondria-Targeted Artificial "Nano-RBCs" for Amplified Synergistic Cancer Phototherapy by a Single NIR Irradiation. Advanced Science. 2018;5(5):1800049.

[101] Pansare V, Hejazi S, Faenza W, Prud'homme RK. Review of Long-Wavelength Optical and NIR Imaging Materials: Contrast Agents, Fluorophores and Multifunctional Nano Carriers. Chemistry of Materials. 2012;24(5):812-827.

[102] Ren F, Ding L, Liu H, et al. Ultra-small nanocluster mediated synthesis of Nd3+-doped core-shell nanocrystals with emission in the second near-infrared window for multimodal imaging of tumor vasculature. Biomaterials. 2018;175:30-43.

[103] Pisanic TR 2nd, Zhang Y, Wang TH. Quantum dots in diagnostics and detection: principles and paradigms. The Analyst. 2014;139(12):2968-2981.

[104] Liu H, Li Z, Sun Y, et al. Synthesis of Luminescent Carbon Dots with Ultrahigh Quantum Yield and Inherent Folate ReceptorPositive Cancer Cell Targetability. Scientific Reports. 2018;8(1):1086.

[105] Pellerin M, Glais E, Lecuyer T, et al. $\mathrm{LaAlO}_{3}: \mathrm{Cr}^{3+}, \mathrm{Sm}^{3+}$ : Nano-perovskite with persistent luminescence for in vivo optical imaging. Journal of Luminescence. 2018;202:83-88.

[106] Schroeder KL, Goreham RV, Nann T. Graphene Quantum Dots for Theranostics and Bioimaging. Pharm Res. 2016;33(10):23372357.

[107] Hai X, Guo Z, Lin X, et al. Fluorescent TPA@GQDs Probe for Sensitive Assay and Quantitative Imaging of Hydroxyl Radicals in Living Cells. ACS Appl Mater Interfaces. 2018;10(6):5853-5861. 